\title{
Spot the difference: Causal contrasts in scientific diagrams
}

\author{
Draft of June 10, 2016
}

\begin{abstract}
An important function of scientific diagrams is to identify causal relationships. This commonly relies on contrasts that highlight the effects of specific differencemakers. However, causal contrast diagrams are not an obvious and easy to recognize category because they appear in many guises. In this paper, four case studies are presented to examine how causal contrast diagrams appear in a wide range of scientific reports, from experimental to observational and even purely theoretical studies. It is shown that causal contrasts can be expressed in starkly different formats, including photographs of complexly visualized macromolecules as well as line graphs, bar graphs, or plots of state spaces. Despite surface differences, however, there is a measure of conceptual unity among such diagrams. In empirical studies they generally serve not only to infer and communicate specific causal claims, but also as evidence for them. The key data of some studies is given nowhere except in the diagrams. Many diagrams show multiple causal contrasts in order to demonstrate both that an effect exists and that the effect is specific - that is, to narrowly circumscribe the phenomenon to be explained. In a large range of scientific reports, causal contrast diagrams reflect the core epistemic claims of the researchers.
\end{abstract}

\section{Introduction}

Biology is littered with visuals. Some of them are well known even in popular culture, for instance the DNA double helix or evolutionary trees. But beyond these iconic representations we find an extensive visual practice of workaday scientific diagrams. They appear copiously in laboratory meetings and conference talks, and almost all publications include them. An indication of the fact that diagrams are essential rather than decorative is that scientists often advise their students to start reading a paper by looking at the figures. In addition to generating understanding, scientific diagrams appear to be linked with core issues in the philosophy of science such as confirmation, explanation and representation.

Philosophers of science have yet to develop an adequate systematic understanding

1

(C) 2016. This manuscript version is made available under the Elsevier user license http://www.elsevier.com/open-access/userlicense/1.0/ 
of scientific diagrams. There exists no standard corpus of works that give a connected view of the functions that scientific diagrams have, how they perform these functions, and how they relate to the established topics in the history and philosophy of science. However, the analysis of scientific diagrams has advanced in the past decade: there is now enough of a literature to frame the debate and to delineate at least some of the key issues that are at stake. ${ }^{1}$

Recently, Bechtel and Abrahamsen (2015) outlined three main functions of diagrams in mechanistic research: diagrams are used, first, to delineate phenomena to be explained; second, to identify causal or explanatory relations between variables; and third, to construct and revise mechanistic models. Even a cursory study shows that each of these functions has many instances in actual science.

The present paper will look in detail at the second of Bechtel's and Abrahamsen's diagram functions, the identification of causal relationships. Many diagrams serve this goal by highlighting difference-makers: they create appropriate contrasts to show changes in an effect under particular interventions. Such diagrams are thus closely tied both to philosophical accounts of causation and to the practice of causal inference. In the 19th century, John Stuart Mill considered the "method of difference" to be the most reliable of his methods of experimental inquiry (Mill, 1843, III.VIII). Its core idea is that if we compare two instances where an effect occurs in one but not in the other, and whose antecedents differ in only one condition, then we can infer that the sole antecedent difference is causally related to the effect. Although it is an early and highly idealized formulation, the method gives a credible first-pass description of many experiments in science. Major philosophical accounts of causation in the 20th century also try to capture the difference-making nature of causes. Arguing for a counterfactual theory of causation, David Lewis writes: "We think of a cause as something that makes a difference, and the difference it makes must be a difference from what would have happened without it. Had it been absent, its effects [...] would have been absent as well" (Lewis, 1974, p. 557). Similarly, probabilistic theories of causality use conditional probabilities to represent difference-making. Christopher Hitchcock (2012): "Probabilistic theories of causation capture [the] notion of making a difference by requiring that a cause make a difference for the probability of its effect." Difference-making is also an important component of the interventionist account of causation. Woodward (2013) writes that "the most natural way of defining the notion of causal effect is in

\footnotetext{
${ }^{1}$ Laura Perini has argued that visual representations express claims, like linguistic representations (Perini, 2005b). Her studies address the role of visual representations in biological explanation (Perini, 2005a) and confirmation (Perini, 2005c). Goodwin (2009) has challenged Perini's view that visual representations are apt for truth and falsity, arguing that they should be understood more along the lines of scientific models (for a reply, see Perini, 2012). A tradition in cognitive science asks how reasoning is facilitated by visual representations (see for instance Larkin and Simon, 1987; Hegarty et al., 1988; Hegarty, 2004, 2011). Finally, the state of the art was advanced in recent years by the Working Group On Diagrams in Science (WORGODS) at the University of California at San Diego. In addition to the discussion in the main text, see Bechtel and Abrahamsen (2012), Sheredos et al. (2013), Bechtel et al. (2014), Burnston et al. (2014) and Burnston (2016).
} 
terms of the difference made to the value of $\mathrm{Y}$ by a change or difference in the value of X" (emphasis in original; see also Woodward, 2003b). Finally, in the mechanistic framework, Craver and Darden (2013) note that mechanisms operate because the entities and activities at one stage make a difference to the entities and activities at a later stage. This leads to a natural view of experimental practice: "Experiments that test for causal relevance test whether a given entity, property, activity, or organizational feature makes a difference to what happens at a later stage in the mechanism" (chapter 8). They then offer a detailed and useful discussion of kinds of difference makers and of the methods used to study them. Thus, the notion of difference-making is widespread both in theoretical accounts of causation and in methodological discussions of causal inference.

How causal contrast diagrams are used to learn and reason about difference-makers will be the subject of four case studies from the life sciences, spanning a wide range of different styles of research. The breadth of cases will show that causal contrasts can be found in many superficially very different scientific diagrams and at many levels of inquiry, including research where mechanistic understanding is not yet in sight.

The first case study concerns the molecular and experimental investigation of RNA interference, where the ubiquity of diagrams showing results from difference-making experiments may be unsurprising (section 2.1). We then proceed to a non-molecular but still experimental case in ecology: Gause's tests of the Lotka-Volterra predator prey model (section 2.2). The third case is neither molecular nor experimental: Doll's and Hill's work on the association of smoking and lung cancer (section 2.3). Finally, the fourth case is a purely theoretical contribution by Alberch on the interaction of development and natural selection in evolutionary processes (section 2.4).

In order to facilitate thinking about causal contrast diagrams, it will be useful to import a number of notions from Woodward's interventionist framework. Among the accounts of causation currently on offer, interventionism is particularly well developed with regard to biological practice (see among others Woodward, 2003b; Waters, 2007; Woodward, 2010; and the already cited Craver and Darden, 2013, chapter 8). A causal relationship between $\mathrm{C}$ and $\mathrm{E}$ will be understood as one where an intervention I on $\mathrm{C}$ results in a change in $\mathrm{E}$ in at least some background circumstances B. Scientists learn about such relationships either by performing the relevant interventions or by searching for "natural experiments" in which $\mathrm{C}$ is changed by an unmanipulated cause $\mathrm{I}^{*}$ that nevertheless has the properties of an intervention - most importantly, $\mathrm{I}^{*}$ must not cause a change in E by any causal paths that do not lead through C (Woodward, 2003a, p. 94; Scheines, 2005). Instances of both types of inferences will be discussed below: section 2.1 (on post-transcriptional gene silencing) and section 2.2 (on experimental tests of the predator-prey model) present cases in which manipulation occurs, while section 2.3 (on the relationship between tobacco consumption and lung cancer) discusses an observational study. Crucially, the study of interventions gives knowledge 
of "partial" rather than "total" causes: interventions demonstrate causal relevance of $\mathrm{C}$ to $\mathrm{E}$ under circumstances $\mathrm{B}$, but other variables will also be relevant to $\mathrm{E}$ either in conjunction with $\mathrm{C}$ or by different causal pathways. It remains for further research to discover the range of background conditions $B$ under which the relationship between $C$ and $\mathrm{E}$ remains invariant. Some relationships will be stable over a small range of invariance, while others will be stable over very wide range (Woodward, 2010, pp. 291-296). Scientists sometimes prize stability: Section 2.1 tells the story of the discovery of a causal relationship that proved to be important but quite unstable; a later, similar discovery of a more stable relationship quickly won its discoverers a Nobel Prize. Finally, causal relationships may be more or less specific in the sense that varying the state of the cause $\mathrm{C}$ allows us to modulate the state of the effect $\mathrm{E}$ in a more or less fine-grained way (Woodward, 2010, 301-314). For instance, we would speak of a specific effect when a carefully targeted chemotherapeutic agent affects only the growth of cancer cells rather than the growth of all cells, as an unspecific spindle poison would. We will see in sections 2.1 and 2.3 that diagrams are often used to demonstrate that causal relationships are specific.

Before we proceed, a note on terminology is in order. The term "diagram" will be understood broadly to include most visual representations in science. For present purposes I exclude only strictly depictive photographs and drawings (e.g. of a bird's plumage). The term will be used to cover not only line graphs, bar graphs, and scatterplots in state spaces, but also photographs that are used to detect (rather than depict) radioactive markers and fluorescent macromolecules.

\section{Causal contrasts in scientific diagrams}

\subsection{Molecular biology: Post-transcriptional gene silencing}

One way to change the activity of a gene is to intervene on the DNA sequence of the gene itself or on the sequence of the promotor regions that regulate the gene's transcription. However, it is usually easier to intervene after transcription has occurred - that is, to intervene on the mRNA transcript of the gene before it is translated into a protein. Thanks to research conducted from the early 1980s and culminating in a Nobel Prize awarded in 2006, it is now a key tool of molecular biology to downregulate specific mRNAs by so-called small interfering RNA. In this section we will look at two publications from the history of so-called post-transcriptional gene silencing in order to see how the effects of small RNAs were established. We will see that diagrams played a crucial epistemic and evidential role.

In the 1980s, a number of researchers explored the effects of specific, singlestranded anti-sense RNAs on the levels of complementary mRNA and its translation.

The anti-sense RNAs were thought to hybridize with the endogenous mRNA and thereby 
either to prevent translation or to elicit the destruction of the double-stranded RNA. A representative instance of this research is the work by Crowley, Nellen, Gomer and Firtel (1985) at the University of California at San Diego.

The authors studied the slime mold Dictyostelium, which has two modes of growth. When grown on bacteria as a food source, Dictyostelium grows as single cells. Upon starvation, however, cells begin to stream together to form multicellular "fruiting bodies". At the molecular level, this process involves the discoidin I family of genes. In mutants of discoidin I, the process of streaming together is disrupted, although cells still form aggregates by random collision.

Crowley et al. showed that the activity of the discoidin I genes can be reduced by RNA that binds to discoidin I mRNA. To demonstrate this, they constructed a plasmid: a short, circular piece of DNA that can be artificially introduced into cells. It encoded a single-stranded anti-sense RNA complementary to a part of the discoidin I- $\alpha$ mRNA. After introducing this plasmid into Dictyostelium, they found that it caused at least a 20-fold decrease in discoidin I mRNA. The diagram supporting this claim is here reprinted as figure 1 .

Briefly, the data in figure 1 was produced as follows. The plasmid encoding antisense RNA was transfected into Dictyostelium together with another plasmid (B10SX) conferring resistance to an antibiotic. In the negative control, B10SX alone was transfected. ${ }^{2}$ The antibiotic was then used to screen for successfully transformed cells, from which colonies were grown. RNA from four colonies was isolated and separated by electrophoresis. The four colonies were: B10SX (the negative control), Rev 7 and Rev 5 (both transformed with the "reverse", anti-sense discoidin plasmid), and Disc/2H3 (effectively another negative control, but see footnote 2). The RNA was then labeled with three different radioactive probes to visualize it on X-ray sensitive film. One probe was specific for mRNA of discoidin I (labeled Discoidin, left panel in the figure); the second for mRNA from another plasmid-encoded gene (labeled Neo, middle panel); and the third for mRNA of Dictyostelium actin (labeled Actin, right panel), a ubiquitous cytoskeletal protein.

We can now see that figure 1 creates three contrasts to establish a causal relationship and to circumscribe its specificity. Contrast 1 shows a marked decrease in discoidin I mRNA in cells transfected with the plasmid expressing anti-sense discoidin I RNA but not in a negative control where no anti-sense RNA is expressed. Two subcontrasts then show that mRNA from a second plasmid-encoded gene (Neo) is not decreased (contrast 2a), nor is endogenous mRNA for Dictyostelium actin (contrast $2 b$ ). This creates an overall contrast 3 showing that the effect on discoidin I mRNA is specific, rather than

\footnotetext{
${ }^{2}$ Dictyostelium was also transfected with a different plasmid, labeled Disc/2H3, that encoded a Dictyostelium gene (in sense direction) and used the same promoter as the anti-sense construct. The researchers used this as a positive control to test that the promoter functioned as expected. For the present discussion, however, the Disc/2H3 construct can be understood as another negative control (it does not express the anti-sense construct).
} 


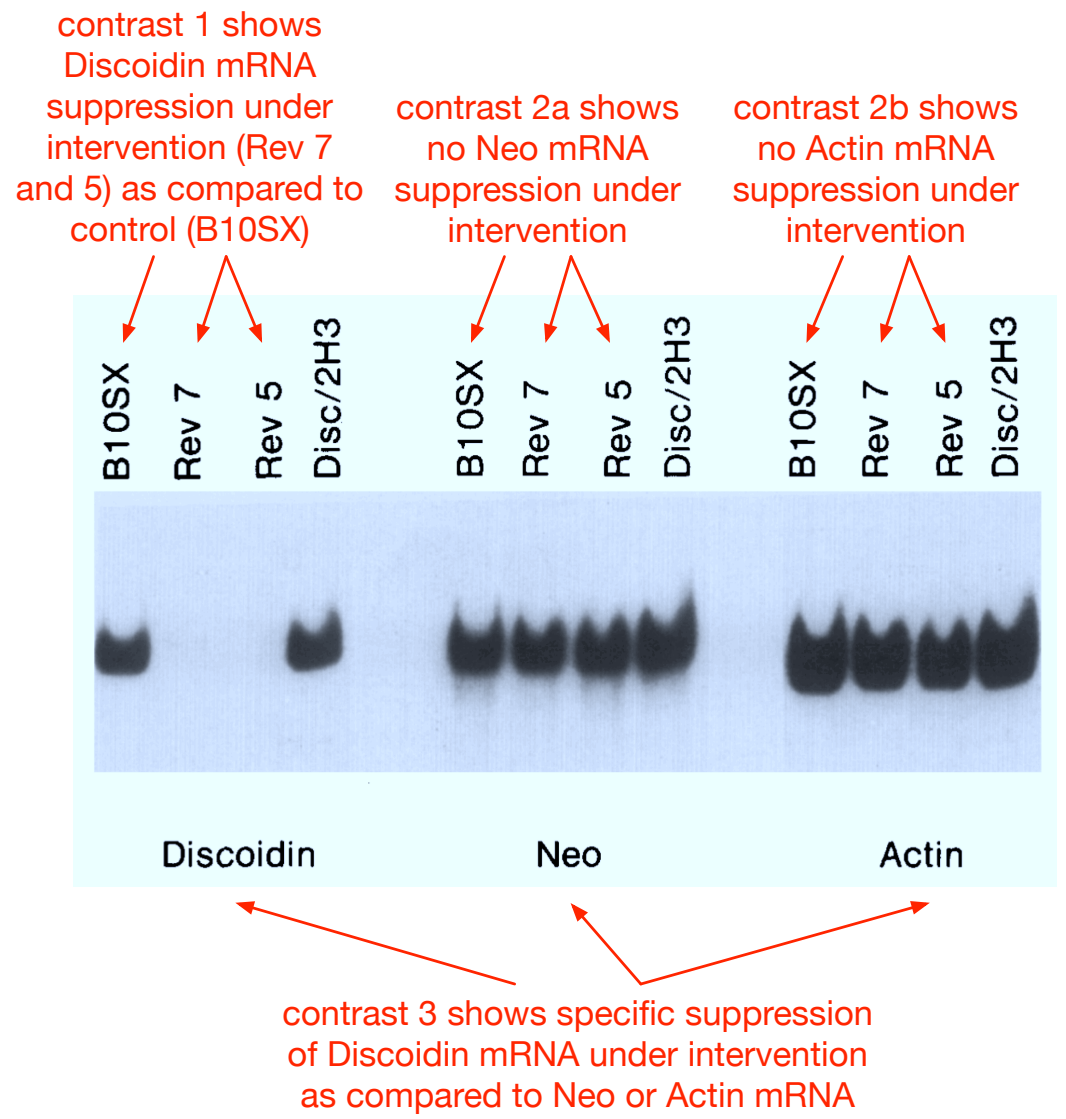

Figure 1. Crowley et al. (1985, p. 635) show that discoidin I mRNA (Discoidin, left) is suppressed by a plasmid expressing complementary anti-sense RNA: note the absence of labeled discoidin I mRNA in two samples from transformed Dictyostelium colonies (Rev 7 and Rev 5). B10SX indicates a negative control without the anti-sense plasmid. Disc/2H3 is a different plasmid construct which we can understand as another negative control (discussed in footnote 2). Importantly, mRNA levels of a different plasmid-encoded gene (Neo, middle) and of Dictyostelium actin (Actin, right) do not decrease. Thus, the diagram creates three contrasts. Contrast 1 shows the effect of anti-sense discoidin I RNA on discoidin I mRNA levels. Contrasts $2 a$ and $2 b$ show that this effect is absent for Neo and Actin mRNA. Thus, the overall contrast 3 shows that the effect is at least somewhat specific to the targeted mRNA and not, for instance, due to a generalized suppression or degradation of mRNA. See text for a detailed discussion. The arrows indicating contrasts are not part of the original figure. 
a generalized effect on mRNA transcription or degradation. Demonstrating that the effect is fully sequence-specific would, of course, require further contrasts.

The researchers went on to create similar contrasts at the organismic level (figure 8 in the cited paper, here not printed). They tested the effects of the plasmid on the formation of streams in Dictyostelium's fruiting body aggregation, finding that the intervention with anti-sense RNA created a non-streaming phenotype similar to discoidin I mutants, a so-called phenocopy.

With the analysis of figure 1 in place, we now proceed to a second diagram from the further history of RNA interference techniques. We will see that the second diagram instantiates many of the same concepts as the first, despite differences in experimental setup and measurement techniques.

Experiments such as those by Crowley et al. demonstrated that interventions with single-stranded RNAs were sometimes effective for post-transcriptional gene silencing. However, they were never sufficiently reliable to become a widely used research tool. In interventionist terminology, the suppression of mRNA by single-stranded antisense RNA was not a particularly stable causal relationship. The breakthrough for RNA interference techniques came in 1998 with a publication by Fire, Montgomery, Kostas, Driver and Mello (1998). Working on the nematode Caenorhabditis elegans, the researchers showed that injecting short, double-stranded RNA (rather than singlestranded anti-sense RNA) created a "potent and specific" (p. 806) interference effect - not only in injected animals but also in their progeny. RNA interference by doublestranded RNA proved to be a stable effect that quickly became a major research tool for exploring gene function (see e.g. Hannon and Rossi, 2004). Andrew Fire and Craig Mello received the 2006 Nobel Prize in Physiology or Medicine for their contribution.

Intriguingly, small numbers of RNAs were sufficient to elicit a strong effect, indicating that the mechanism of action did not involve simple one-to-one base-pairing between exogenous and endogenous RNAs. Two years later, part of the responsible mechanism was elucidated by Hammond et al. (2000) at Cold Spring Harbor, who discovered that double-stranded RNAs activate a sequence-specific nuclease that uses the exogenous RNAs as guides to homologous transcripts.

Figure 2 reprints what is arguably the key diagram (out of three) in the 1998 paper. It shows the effect of double-stranded RNA (dsRNA) on the expression of green fluorescent protein (GFP) in the progeny of injected animals. The GFP protein is not native to $C$. elegans: The strain used for these experiments was a so-called reporter strain engineered to express GFP, which can be conveniently visualized with appropriate microscopes. Now observe the contrasts: In the control with the double-stranded RNA ds-unc $22 A$, which targets a muscle protein, GFP is expressed at normal levels in both the nuclei and the mitochondria of both the larva (a) and the adult (b). When dsRNA targeting GFP is introduced (ds- $g f p G$ ), GFP disappears almost completely in the larva (d) and the adult (e). The exception is the vulval musculature (see e), which 


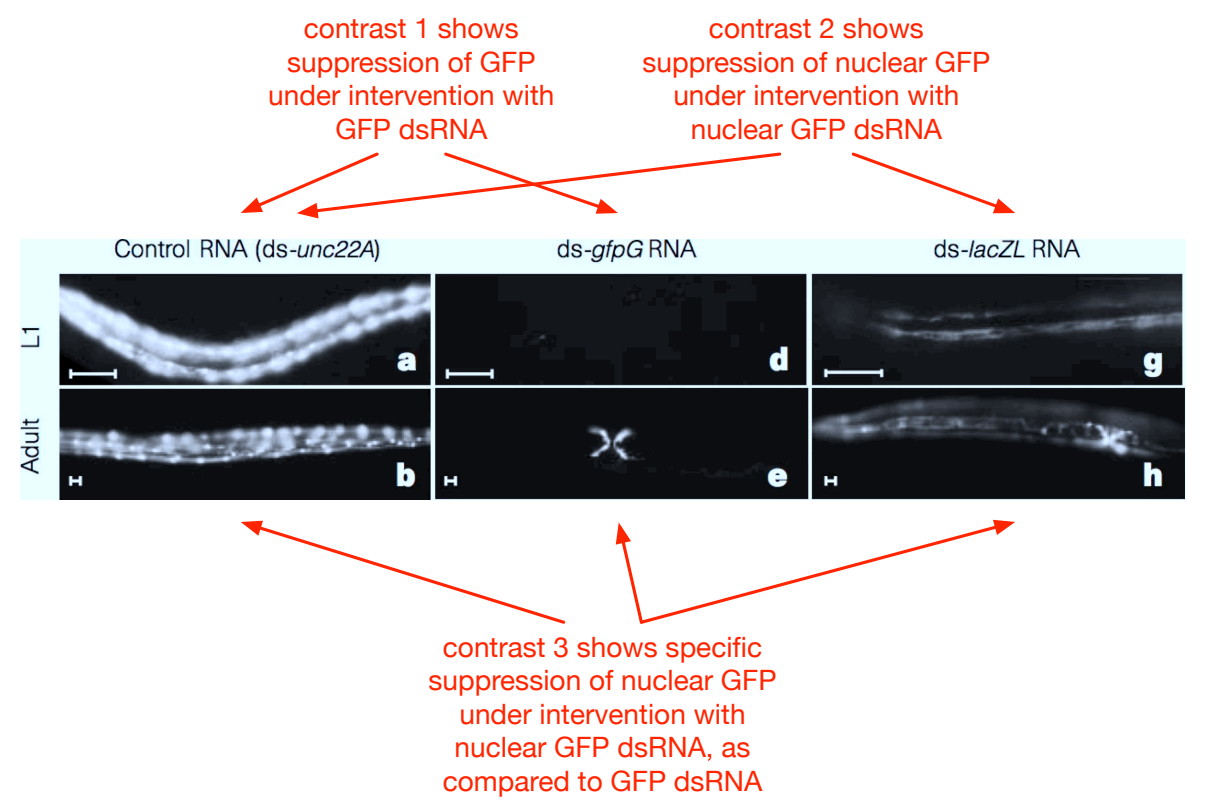

Figure 2. Fire et al. (1998, p. 808) demonstrate the effect of double-stranded RNA (dsRNA) on the expression of nuclear and mitochondrial green fluorescent protein (GFP) in the progeny of injected C. elegans. In panels $\mathrm{a}$ and $\mathrm{b}$ (control), GFP is fully expressed in the larva and adult, respectively. In $\mathrm{d}$ and e (treatment with dsRNA homologous to GFP mRNA), GFP is fully suppressed in the larva in d. In the adult in e, only the non-striated vulval muscles (cross-shaped), which originate late in development, escape the GFP suppression effect. In $g$ and $\mathrm{h}$ (treatment with dsRNA targeting only nuclear GFP), nuclear GFP disappears while mitochondrial GFP remains. Again three contrasts are highlighted. The first contrast demonstrates the effect of GFP dsRNA on GFP, the second demonstrates the effect of nuclear GFP dsRNA on nuclear GFP, and the third demonstrates that the suppression is specific. The original figure contains magnifications in three additional panels $(\mathrm{c}, \mathrm{f}$ and i) which are here omitted for clarity. See text for a detailed discussion. The arrows indicating contrasts are not part of the original figure. 
originates late in larval development and escapes the interference effect of the dsRNA injected into the parent. Otherwise cells expressing GFP are rare (f). When dsRNA targeting only nuclear (but not mitochondrial) GFP is introduced (ds-lacZL), only the nuclear signals disappear (larva: g, adult: $\mathrm{h}$ ).

Despite surface differences, figure 2 is thus conceptually similar to figure 1 . Both establish a first contrast between control and intervention to show that the intervention has an effect - small RNAs reduce GFP mRNA levels. A second contrast then shows that the cause can be targeted precisely: if a part of the sequence that is specific to nuclear GFP is targeted, then only nuclear GFP disappears. This creates a third, overall contrast which shows specificity: RNA transcription is not suppressed in general, and suppression may well be limited to the targeted nucleotide sequence (although only further experiments could show this). We can see here that the same diagrams serve two known functions in the account by Bechtel and Abrahamsen (2015). First, they establish a causal relationship, but second, they also specify the phenomenon, or effect, that is to be explained in the first place: sequence-specific rather than generalized mRNA suppression. The use of two contrasts serving these particular functions is a recurring feature of causal contrast diagrams, as section 2.3 will show.

We have seen two diagrams that create contrasts to highlight the specific effects of difference-makers. They serve multiple purposes. One is straightforwardly communicative: the contrasts convey newly discovered causal relationships to readers. But the diagrams also have evidential and epistemic roles. The diagrams are evidence for facts about differences that occur under particular interventions. On the basis of that evidence, we then infer causal relationships: that interventions on a variable ("presence of dsRNA homologous to mRNA of gene X") result in changes of another variable ("suppression of gene X's product"). In this process, the diagrams are key material intermediates between a series of manipulations in the laboratory and the epistemic conclusions that scientists aim for.

Translating diagrams of this sort into linguistic propositions, while possible in principle, would greatly reduce the readers' access to the evidence for the paper's claims. In a court of law, the difference is that between declaring "a bloodstained knife was found at the murder scene" and actually producing the knife in question along with photographs showing its context at the crime scene. There is a distinction to be made between providing physical evidence and providing a verbal description of that physical evidence. Even if the verbal representation were entirely accurate and complete, the key evidence would still be the knife (or the electrophoresis gel) that the description refers to, not the description itself. ${ }^{3}$

\footnotetext{
${ }^{3}$ I am here not challenging Perini's view that diagrams can themselves have propositional content (Perini, 2005b, 2012; and see Goodwin, 2009, for an opposing viewpoint). The point is only that in cases where diagrams serve as material evidence, translating them into verbal representations would remove an important part of the warrant for the conclusions we base on them.
} 


\subsection{Ecology: Testing the predator-prey model}

The Russian biologist Georgii Frantsevich Gause was among the first to put Vito Volterra's predator-prey model to empirical tests. The presentation of his results in Gause (1934b, chapter 6) relies heavily on diagrams, of which we will consider a representative instance.

Volterra $(1926,1928)$ had proposed a mathematical model for the interaction of a prey and a predator species. ${ }^{4}$ The model assumed that the prey would grow exponentially in the absence of a predator, while the predator would die exponentially in the absence of prey. The two species were also linked, such that the predator population grew in proportion to the prey population while, conversely, the prey population diminished as the predator population increased. These model properties and relationships are represented by the following pair of coupled differential equations

$$
\begin{aligned}
& \frac{d N_{1}}{d t}=\left(\varepsilon_{1}-\gamma_{1} N_{2}\right) N_{1} \\
& \frac{d N_{2}}{d t}=\left(-\varepsilon_{2}+\gamma_{2} N_{1}\right) N_{2}
\end{aligned}
$$

where $N_{1}$ is the size of the prey population, $N_{2}$ is the size of the predator populations, $\varepsilon_{1}$ and $\varepsilon_{2}$ are their respective growth rates, and $\gamma_{1}$ and $\gamma_{2}$ are coefficients that tell us how well the prey species defends itself against the predators and how much the predators profit from eating the prey.

Under appropriate parametrization, the predator-prey model can produce population oscillations such as are observed in nature. For Volterra and many of his readers, this counted as one of the model's main explananda. Volterra (1928) devoted a figure to it in his text, here reprinted as figure 3. It is easy to grasp the interaction of the two populations: the growth of the prey population $\mathrm{N}_{1}$ allows the predator population $\mathrm{N}_{2}$ to grow as well, until the number of predators is so large that the prey population begins to decrease. This in turn causes the predator population to decrease for lack of sustenance. Then the growth of the prey population begins anew.

Gause was sensitive to the fact that the mere agreement between model prediction and observation did not show that the true causes of population oscillations had been found. He explained the methodological reasons for this as follows:

If we were asked what proof there is of the fact that the biological system consisting of predator-prey actually possesses "innate" periodic oscillations in numbers of both species [...] we can give but one answer: observation under natural conditions are here of no use, as in the extremely complex natural environment we do not succeed in eliminating "induced"

\footnotetext{
${ }^{4}$ Since Alfred Lotka et al. (1925) pursued a similar mathematical approach to modeling biological phenomena around the same time, we now refer to the Lotka-Volterra model. Gause was aware of and cited Lotka's work.
} 


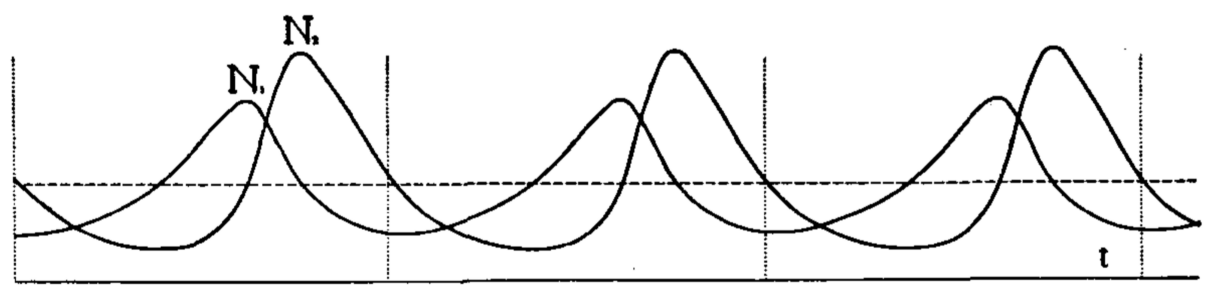

Figure 3. Volterra (1928) plots the oscillations in the population sizes of a prey species $\left(\mathrm{N}_{1}\right)$ and predator species $\left(\mathrm{N}_{2}\right)$ under appropriate parametrization of his model. Population sizes are plotted on the vertical axis, time on the horizontal. This is not a causal contrast diagram, but it is the basis for the causal contrasts shown in figure 4.

oscillations depending on cyclic changes in climatic factors and on other causes. (Gause, 1934b, p. 117)

In order to separate confounding causes such as climate from the internal dynamics of the predator-prey system, Gause found investigations "under constant and exactly controlled laboratory conditions" indispensable (p. 117). Thus, he constructed an experimental system consisting of two unicellular organisms. The prey was Paramecium caudatum, living on bacteria in the culture medium; the predator was Didinium nasutum, whose diet consists mainly of Paramecium.

Gause used a diagram to summarize his main experimental results on predator-prey dynamics, which is here reprinted as figure 4. Like Volterra's and D'Ancona's figure, the diagram plots the number of the predator and prey species on the vertical axis and time on the horizontal axis. The difference is that Volterra plotted the predictions of the mathematical model, while Gause plotted empirical data to test said mathematical model.

Gause's figure has three panels, which correspond to his three main findings. The top panel shows that the predictions of the mathematical model are not met for a simple predator-prey systems. If predators are added to the culture, they will grow, devour the prey, and then perish themselves - even if the prey population was already well established. Thus, the end result is a culture consisting only of the bacterial base of the food chain. The predicted innate oscillations could not be found. Gause diagnosed the problem to a specific model assumption: the function linking the predator population size to the prey population size. According to the model, as the prey diminishes the predators will diminish proportionately, thus allowing the cycle to restart. In fact, however, Gause observed that low numbers of Paramecium did not cause Didinium to perish proportionately. Instead, the Didinium organisms kept dividing, albeit at a smaller size, and thereby effectively increased their "seizing surface" (p. 121) until all of the prey were gone. ${ }^{5}$

The middle panel shows what happens if the culture medium is made heterogeneous

\footnotetext{
${ }^{5} \mathrm{~A}$ later experiment succeeded in reproducing Volterra's periodic oscillation and was reported in Gause (1935) as well as later in chapter 6 of Gause (1934b).
} 


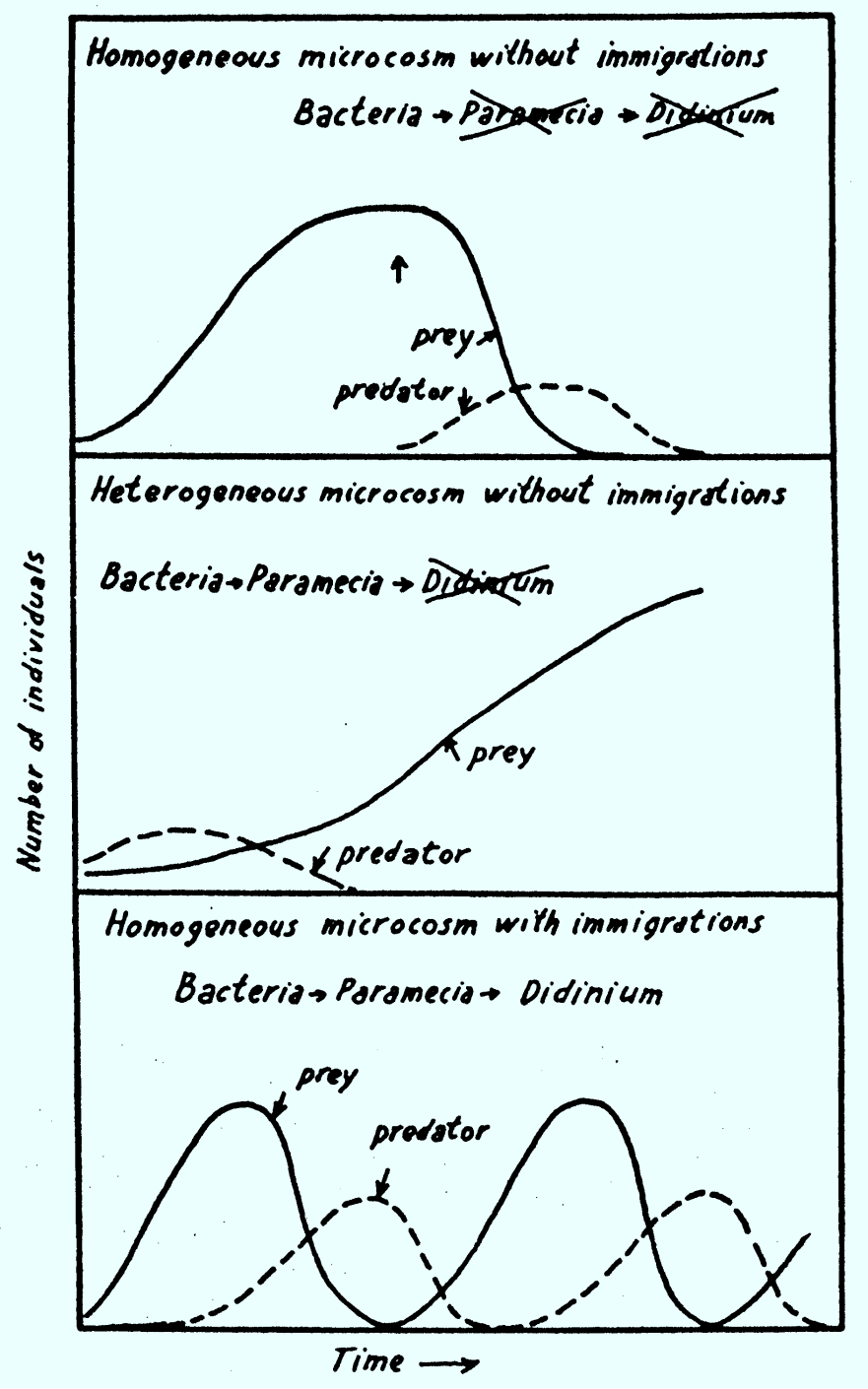

Figure 4. A key figure from Gause (1934b, p. 127) summarizes experiments on a predator-prey system consisting of the protists Paramecium caudatum and Didinium nasutum. Top panel: The internal dynamics of the predator-prey system do not show the oscillations predicted by mathematical theory. Instead, the predator eats the prey and then die off. Middle panel: The addition of refuges for the prey permits the prey to dominate the culture after the predator has died out. Bottom panel: The periodic immigration of predators and prey into the culture produces oscillations. Notice that the middle and bottom panels each contrast with the top panel, highlighting the effects of refuges and immigrations, respectively. See text for details. 
by adding refuges for the prey. These were provided by a "dense oaten medium" with sediment (p. 121), in which the Paramecia were known to be safe from Didinium. In this case, the predators will devour all the prey except for those hiding in the refuges. After the predators have died out for lack of food, the prey can repopulate the entire culture, which thus ends as a mixture of bacteria and Paramecia. However, Gause found that this is only one of multiple possible outcomes "because the struggle for existence is here affected by a multiplicity of causes" (p. 123). In some cultures, the prey were entirely devoured despite the refuges; in others, the situation depicted in the middle panel obtained; and in some, the continued influx and outflux of prey into the refuges allowed predators and prey to coexist for a time.

In the bottom panel, the culture is again homogeneous, but immigrations of both species are arranged at regular intervals (although the number of immigrating organisms is relatively small relative to the total population sizes). This produces oscillations comparable to those observed in nature. The culture reaches a state where bacteria, Paramecia and Didinium co-exist.

To summarize, the contrasts between the three panels of figure 4 permit us to see the effects of two interventions on predator-prey dynamics: refuges and immigrations. Pure predator-prey interactions (as assumed by theory) lead to the extinction of both species. This contrasts with inhomogeneous cultures containing refuges, which can allow the prey to end up dominating the culture. It further contrasts with homogeneous cultures with immigrations, which can permit oscillations as observed in nature. Both refuges and immigrations are thus identified by intervention as difference-makers that affect predator-prey dynamics: they must be part of at least some accurate models of the phenomenon.

Given the multiple paragraphs it takes to explain the content of the figure, one might object that the text is doing more work than the diagram. However, the objection mistakes the preparation of Gause's target audience. Recall that the layout of Gause's figure precisely mirrors Volterra's. Once readers are trained to understand the basics of this type of figure, the minimal text in the figure itself goes a long way towards enabling a reader to understand and to compare the different outcomes. As in the previous case in section 2.1, a good deal of technical background knowledge is required to understand the contrasts presented in the figure.

We again find that the figures in Gause's text not only serve for effective communication, but also play a crucial evidential role. In the main text, most of the empirical data is not given at all: The diagrams are the only source of the actual population sizes at different times during the experiments. Although the contrastive summary diagram reprinted here as figure 4 does not give quantities, it combines three other diagrams from earlier in the same chapter which do. These have quantitative scales and plot data points precisely. If one wished to skip the diagrams and look for the raw data elsewhere, one would need to consult the appendix of tabular data at the end of the book, 
but these tables are far from complete. The same practice is even more pronounced in Gause's papers on the same topic, where the figures have quantitative scales but no tabular data is provided (e.g. Gause, 1934a, 1935). Thus, Gause uses the diagrams in a way that makes them evidentially indispensable.

We thus see that the figures discussed in section 2.1 and Gause's figure are conceptually related. Again the diagram creates causal contrasts that directly reflect a key epistemic concern of the research project: in this case, the empirical determination of conditions that allow a predator-prey system to manifest oscillations. In addition, the diagram again has an additional and indispensable evidential role, since it represents population sizes - empirical quantities that are not given in the text.

\subsection{Epidemiology: Smoking causes lung cancer}

In the early 1950s, Richard Doll and Austin Bradford Hill published a series of papers investigating the link between smoking and lung cancer $(1950 ; 1952 ; 1954)$. These are among the most famous and significant observational, population-level studies of the 20th century. Even though they are quite different in subject matter and approach from the two cases we have considered so far, they again employ appropriate contrasts in diagrams in order to make salient and to support key causal claims.

Doll and Hill (1954) present a prospective study using data collected from questionnaires sent to UK medical professionals. The paper contains a total of six tables, which give the paper's main numerical results. A single chart, here reprinted as figure 5, represents the most important contrasts in the data. ${ }^{6}$ Unlike the previous two cases, this one involves no manipulation: the goal is to take advantage of naturally occurring differences in a variable (levels of tobacco consumption) in order to infer its effects on another variable (changes in levels of lung cancer). The causal inference literature recognizes that such observational causal inferences are possible in the absence of manipulation, provided that the naturally occurring change in the putative cause qualifies as an "intervention" in the technical sense (Woodward, 2003a, p. 94; Scheines, 2005; and see the introduction to this paper). While the study of 1954 does not meet the stringent criteria for an observational causal inference that is equivalent to an experimental one, it manages to inch closer to the goal of interpreting the observed association causally. One of the study's key advantages is that it is prospective rather than retrospective: smoking habits are assessed before lung cancer is diagnosed. It can thus be excluded, for instance, that lung cancer increases the desire to smoke, or that

\footnotetext{
${ }^{6}$ Although the graphical summary appears to us as an obvious step, such presentations are a recent innovation. We find that many common forms of graphical representation originated or developed in the 19th century, but they were slow to gain acceptance (Funkhouser, 1937; Friendly, 2008). Less than a century before Doll and Hill, Ignaz Semmelweis's Etiology of Childbed Fever (1861) presented its findings on differences and trends in mortality rates exclusively in tabular form, despite their suitability for graphical representation (Scholl, 2013). Bechtel and Abrahamsen (2015) cite Wunderlich's (1868) pioneering work on daily oscillations in body temperature as another instance of the 19th century preference for tables.
} 
lung cancer patients simply recall their smoking habits as more severe than those who do not have the disease.

Figure 5 shows six instances of the same type of two-dimensional graph. On the horizontal axis of the graphs, we see four groups which we may refer to as nonsmokers, light smokers, medium smokers and heavy smokers. On the vertical axis we see the ratio of observed deaths to expected deaths for each group (where the expectation is that total deaths are distributed equally to all groups, in proportion to their size). Each of the six graphs shows this data for a different outcome: lung cancer, other cancers, other respiratory diseases, coronary thrombosis, other cardiovascular diseases, and all other diseases.

The chart makes two contrasts jump out at the reader. The first contrast concerns the difference in deaths from lung cancer between non-smokers, moderate smokers and heavy smokers. We can recognize smoking as a potential cause of lung cancer thanks to the progression from a low number of deaths among non-smokers to an intermediate number in moderate smokers to a high number among heavy smokers. ${ }^{7}$ The second contrast, comparing the outcomes in multiple disease classes, shows us that lung cancer departs farther above and below baseline than other diseases. This is similar to the use of diagrams in molecular biology that we saw in section 2.1: In combination, the two contrasts are used to argue that lung cancer is an effect of smoking, and that this effect is at least somewhat specific - except for a smaller effect on coronary thrombosis (see below), smoking does not equally affect other diseases.

A visually striking feature of the chart is its use of the ratio of observed to expected deaths on the vertical axis, instead of absolute numbers or percentages. This allows us easily to see the relevant effect - the increase in lung cancer in heavy smokers - as a departure from baseline. In a sense this displays quantitative information in almost qualitative terms, as "effect or no effect": marked departures from baseline register at a glance. ${ }^{8}$ Nevertheless, much quantitative information is still provided, since each bar is labeled with the numbers that underlie the ratio it plots (in the highest bar, for instance, 13 observed cases of cancer vs. an expectation of 7.3, giving a ratio of 1.78). The visual presentation of the data thus does not come at the cost of precision - instead, the visual information helps to contextualize the numerical data by facilitating appropriate comparisons. Doll and Hill themselves believed that figure 5 is a superior presentation of their data than numerical tables, writing that the "distinction between the systematic variation in the mortality from lung cancer with the amount smoked and the irregular (or small) variation observed in the other disease groups studied is perhaps shown more clearly in the Chart" (p. 1453).

\footnotetext{
${ }^{7}$ The authors make special note of the fact that tests for statistically significant differences between groups do not take account of the "biologically important" (p. 1453) progression, which in the terminology of Mill would be described as concomitant variation (1843, III.VIII.\$6). To correct for this they also apply a test for the significance of trends.

${ }^{8}$ Burnston (2016) also emphasizes the ability of diagrams to highlight qualitative information.
} 


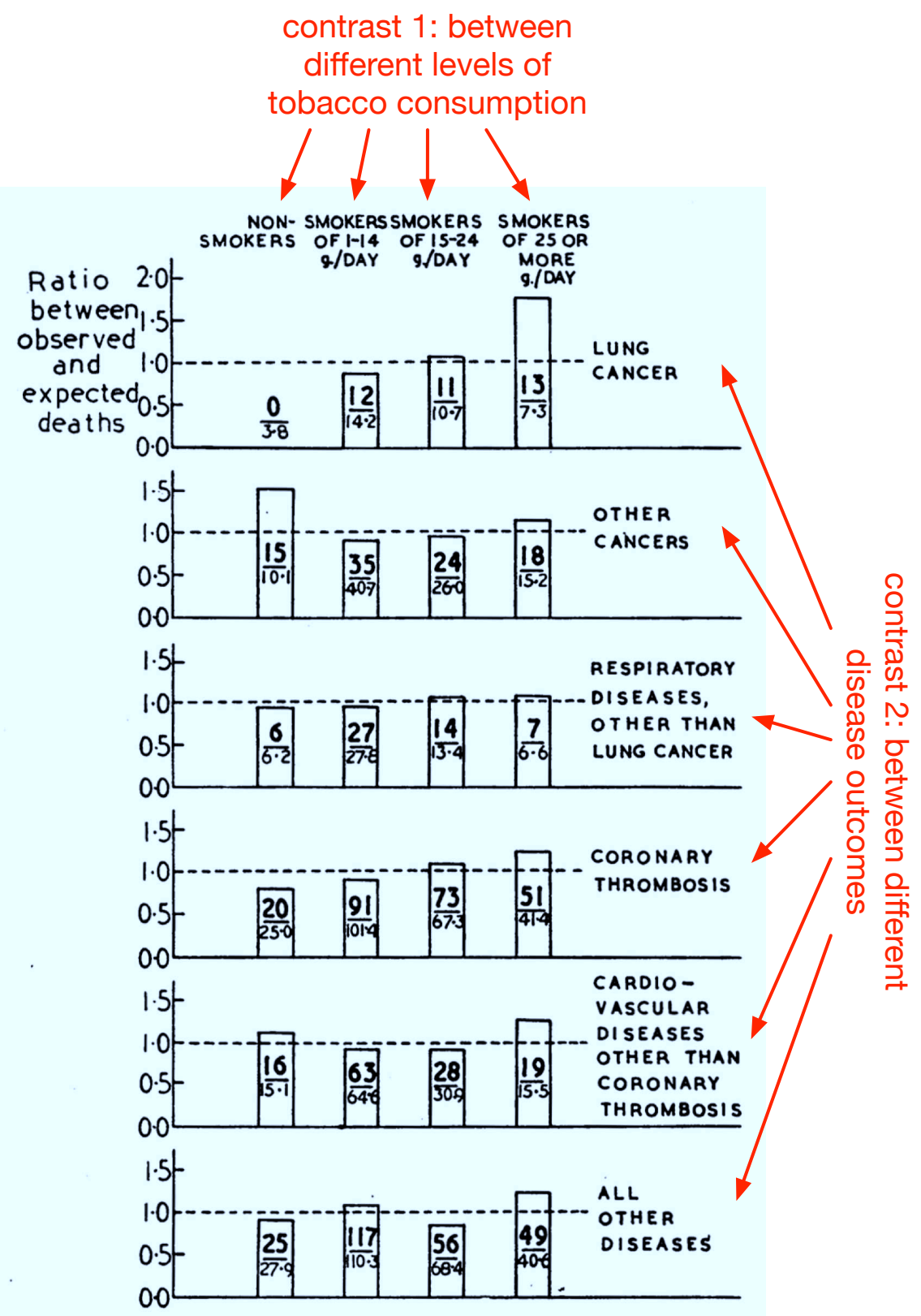

Figure 5. A chart from Doll and Hill (1954, p. 1453) shows two main contrasts. First, an increase in the consumption of tobacco is associated with an increase in lung cancer (contrast 1). Second, the association is strongest with lung cancer, weaker with coronary thrombosis, and not found with other diseases (contrast 2). See text for a detailed discussion. The arrows indicating contrasts are not part of the original figure. 
A virtue of the diagrammatic presentation is that it brings out a number of contrasts in the data in addition to the link between smoking and lung cancer. For instance, in the "other cancers" group, non-smokers appear to die more from cancer than any of the smoking groups. Similarly, under cardiovascular diseases other than coronary thrombosis, it would appear that light and moderate smokers enjoy a benefit over non-smokers. Yet the statistical analysis did not reveal any of these differences to be significant (p. 1453). By contrast, coronary thrombosis clearly suggests a trend of increasing death rates in heavier smokers. In this case, the statistical analysis revealed the trend (not the differences between groups) to be significant, although the authors do not draw any strong conclusions (p. 1453, second footnote). Thus the diagram not only serves to demonstrate the main causal claim of the paper, but it also suggests additional hypotheses that are worthy of exploration.

Beyond clarity of presentation, the diagram's function again includes an evidential component. The only place to find the actual numbers of deaths observed for each group and each disease class is in the diagram. We are given this information in tabular form for only one disease: lung cancer (see the unlabeled table on p. 1453). Similarly, the expected numbers of deaths for each group and each disease class (on which the calculation of the key ratio of observed vs. expected cases is based) are only given in the diagram. The authors' derivation of these expectations (as discussed on pp. 145253 of the paper) relies on age-stratified numbers of deaths that are not provided to the reader except for the case of lung cancer. For the other disease classes, the tables only give annualized death rates per group for all men older than 35 years. ${ }^{9}$ Strikingly, then, a significant part of the raw empirical data of the study is given only in the diagram.

Thus, the chart in Doll's and Hill's 1954 paper expresses the whole set of relevant causal contrasts in the dataset: an effect of smoking on lung cancer, a possible effect of smoking on coronary thrombosis, and no detectable effect on other disease classes. As in the cases discussed before, the diagram reflects the core epistemic claims of the report (changes in levels of tobacco consumption result in changes in lung cancer rates) while also providing otherwise unavailable evidence for them.

\subsection{Evolutionary biology: Development and natural selection}

Pere Alberch was one of the pioneers of evolutionary developmental biology. In the 1980s, he published, together with his collaborator Emily Gale, a landmark empirical study of developmental constraints on natural selection, using the salamander species Ambystoma mexicanum and Hemidactylium scutatum as model organisms. Alberch and Gale (1985) argued that differences in the bone structure of the feet of the two species did not need to be attributed to the action of natural selection. Rather, the differences fol-

\footnotetext{
${ }^{9}$ Together with a table of group sizes, this allows us to approximate some of the original data, since annualized death rate $\times$ group size $\times$ study duration gives the numbers of deaths observed.
} 
lowed as a developmental consequence of different sizes of limb buds, and thus as a side-effect of selection on body size.

Alberch and Gale demonstrated this experimentally by intervening on the limb bud of the developing A. mexicanum embryo with colchicine, which inhibits mitosis. The paper summarizes this key difference-making result in a diagram here reprinted as figure 6. On the left-hand side, the intervention variable is given: the number of cells of the limb bud of A. mexicanum. Two arrows in the middle indicate the control experiment (the normal number of cells, $\mathrm{N}$ ) and the intervention experiment (a reduced number of cells, $\mathrm{n}<\mathrm{N}$ ), respectively. On the right-hand side, we see the two outcomes: the limb bud of normal size produces the bone morphology of A. mexicanum; the limb bud of reduced size produces a different bone morphology. The experimentally produced morphology shows a reduction in both digits and phalanges which is similar to the morphology of $H$. scutatum.

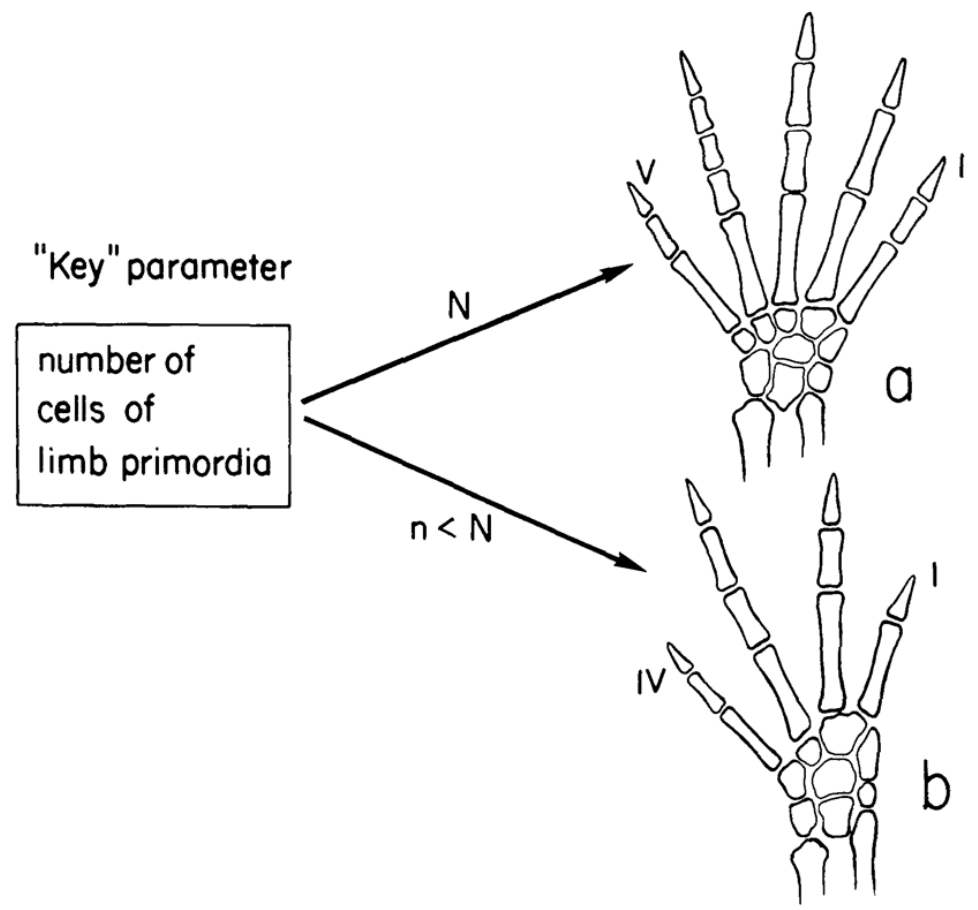

Figure 6. Part of figure 7 from Alberch and Gale (1985, p. 17). It summarizes the key difference-making result of the study: the A. mexicanum embryo with a limb bud of normal size develops into its typical foot morphology (a), while reducing the number of cells in the limb bud with colchicine produces a different morphology similar to H. scutatum (b). Note the loss of a digit and the reduction in phalanges.

This use of a diagram to show an experimental causal contrast is reminiscent of their use in the previously discussed empirical studies. More surprisingly, however, diagrams of causal contrasts also appear in Alberch's earlier theoretical work that has no immediate empirical target. Alberch (1982) sets the agenda for the empirical project by explaining what is at stake in the exploration of developmental constraints. His 
argument is intertwined with a series of diagrams that clearly outline the causal claims at issue.

Two key diagrams from Alberch's theoretical discussion are here reprinted as figures 7 and 8. In figure 7, Alberch invites us to imagine a two-dimensional state space in which we plot two traits $\mathrm{X}$ and $\mathrm{Y}$ of a population of organisms (in lieu of an $n$ dimensional state space for $n$ traits). This might reveal a distribution consisting of multiple clusters (in this case A to D) around certain values of X and Y. In figure 8, Alberch now asks what would happen to population $\mathrm{A}$ if it were (1) raised in an environment of low selective pressure (ample food, no predators, and so on) and (2) exposed to a mutagen. Alberch considers two possible outcomes as $\mathrm{H}_{1}$ and $\mathrm{H}_{2}$. In $\mathrm{H}_{1}$, the variation produced by organisms is "isotropic" (to use a helpful term suggested by Gould, 2002, p. 144-146): it is equally likely to occur in any direction, and so a mutagen in the absence of selective pressures will allow organismic traits to spread out homogeneously in the state space, although clustered around the initial position. The implication is that if traits in natural populations are not spread homogeneously throughout the state space, this is because natural selection has acted on them. By contrast, in $\mathrm{H}_{2}$ the variation produced is structured even in the absence of natural selection. The mechanisms of biological development are assumed to be such that variational processes, unconstrained by selection, will produce recognizable clusters to begin with: notice that clusters A to $\mathrm{D}$ are recreated in addition to previously missing ones. Selection will then favor the clusters that are more advantageous, but the clusters we observe in nature - introduced in figure 7 - are defined both by selection and by the structure of the variation on which selection acts.

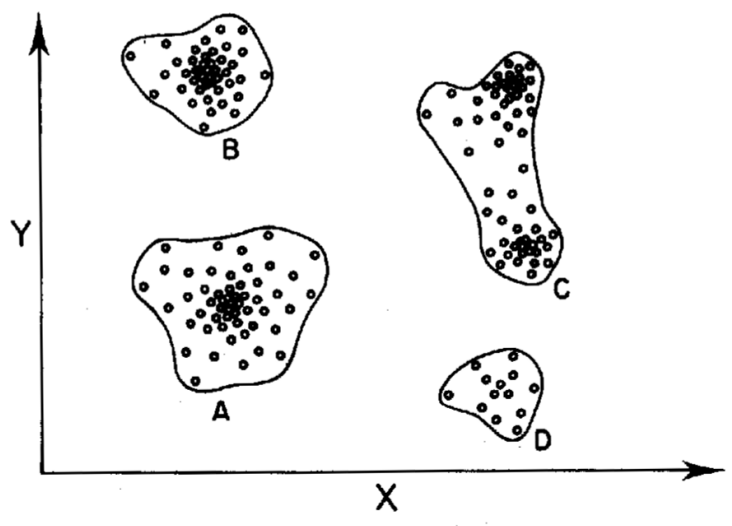

Figure 7. A figure from Alberch's theoretical paper of 1982 (p. 316). Alberch considers a state space in which two phenotypic traits, $\mathrm{X}$ and $\mathrm{Y}$, are plotted for a number of organisms. Alberch assumes clusters $\mathrm{A}$ to $\mathrm{D}$ around four combinations of values for $\mathrm{X}$ and $\mathrm{Y}$. Continued in figure 8 .

Thus, figure 8 again creates a visual contrast in order to represent a claim about difference-making. The hypothetical difference is made by the structure of develop- 


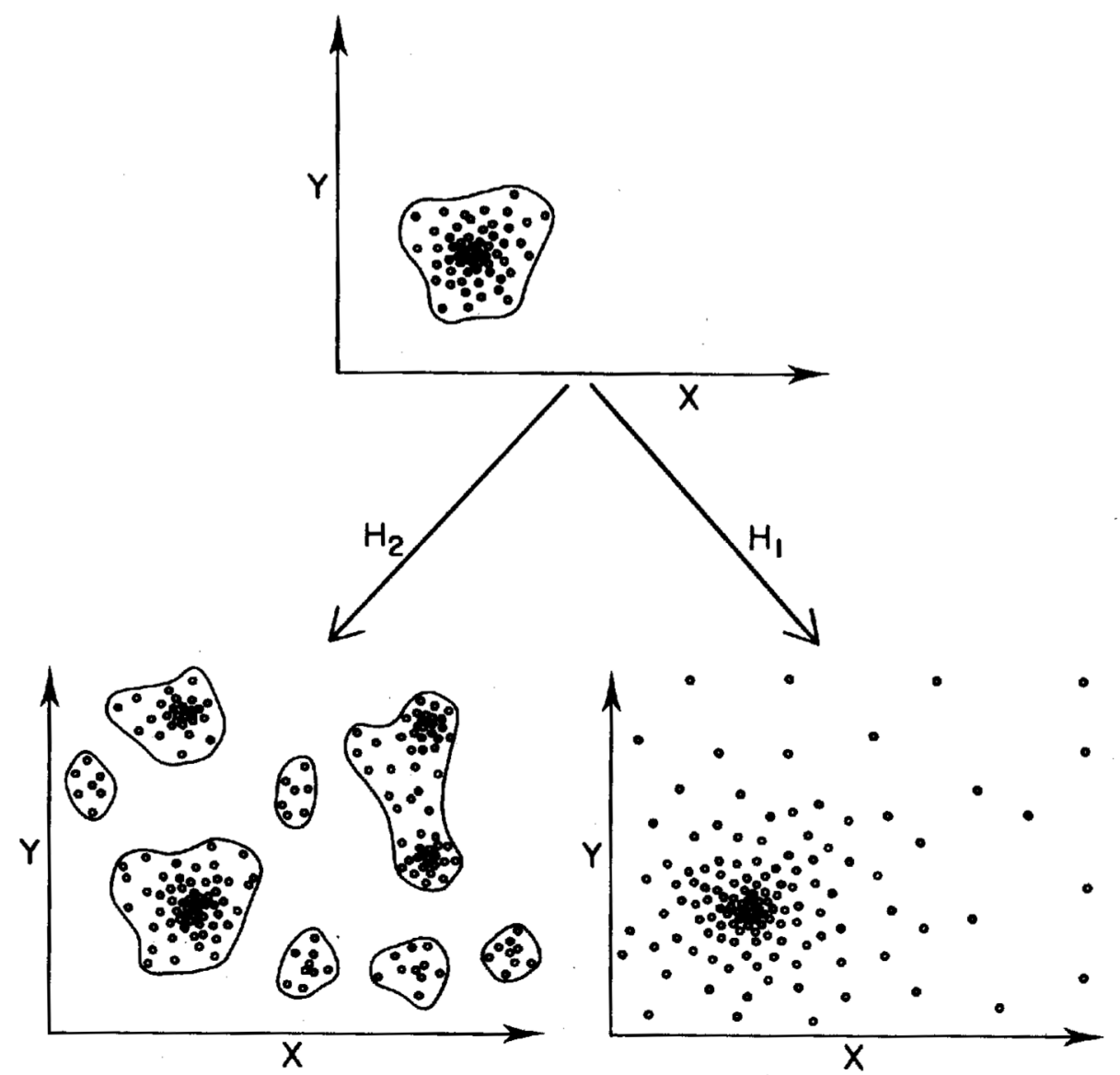

Figure 8. Cluster A from figure 7 is placed in an environment with low selection pressures and exposed to a mutagen (Alberch, 1982, p. 318). Will the traits vary homogeneously around the initial cluster $\left(\mathrm{H}_{1}\right)$, or will they vary in clusters that are defined by the mechanisms of development $\left(\mathrm{H}_{2}\right)$ ? Note that clusters A to D from figure 7 are recreated in $\mathrm{H}_{2}$ along with additional clusters. The contrast between the two hypotheses highlights the effect of a cause - the structure of developmental variation - in evolutionary processes. See text for a detailed discussion. 
mental variation as it enters into the process of natural selection. ${ }^{10}$ If variation is isotropic (as in $\mathrm{H}_{1}$ ), then the structure of the phenotypic variation we observe in natural populations is mainly explained by a the differential survival of some of the variants. If variation is non-isotropic (as in $\mathrm{H}_{2}$ ), then the structure of phenotypic variation must be explained by a combination of developmental mechanisms (which generate nonisotropic variation) and differential survival. Using the figure, Alberch makes these two theoretical causal stories plain.

Notice that both of Alberch's diagrams use bifurcating arrows to indicate the difference that particular causes make: in figure 6 it is the role of limb bud size in determining morphology, and in figure 8 it is the role of the structure of variation in natural selection. Bifurcating arrows appear to be one of the most common ways of representing such information when the different outcomes are discrete, rather than quantitative as in the case of Gause in section 2.2 or Doll and Hill in section 2.3.

Alberch's 1982 paper is at the extreme theoretical end of a spectrum. Unlike the cases discussed previously, it is neither experimental nor observational. Nevertheless, we have seen that a key aspect of his diagrams is conceptually analogous to the earlier cases: visual contrasts in the diagram are used to convey information about the roles of difference-makers. In doing so, the diagrams again represent the key epistemic concerns of the project.

\section{Conclusions}

On the surface, the diagrams discussed in this paper are disparate. We have seen photographs of complexly visualized macromolecules derived from experimental studies; line graphs representing the time course of population sizes under different interventions; bar graphs of observational data on disease occurrence; and finally scatterplots of the distribution of phenotypic variation in a theoretical state space. Nevertheless, all of these diagrams are unified by their use of visual contrasts to highlight the role of difference-makers in the processes under study - in other words, to point to or demonstrate cause-effect-relationships. In many cases, diagrams thus reflect the chief epistemic goals of the research reports in which they appear: they serve to determine causation.

The most obvious way in which diagrams serve this epistemic function is by making relevant contrasts in the data easy to see and understand. ${ }^{11}$ It is an empirical ques-

\footnotetext{
${ }^{10}$ Notice that the present discussion does not presuppose that natural selection as a whole is also a cause in the sense of Woodward (2003b) or a mechanism in the sense of Craver and Darden (2013). Causal roles are determined for more narrowly defined variables: for instance, we ask whether interventions on developmental mechanisms or on fitness values (or on both) would cause organisms to appear in a previously empty part of the phenotype space.

${ }^{11}$ Causal contrast diagrams are related to the graphic design technique of "small multiples", where a series of small and similar displays are shown together (see Edward Tufte, 1983, pp. 170-174). Differences between displays can then be used to highlight change or causality. This facilitates "local comparisons within
} 
tion far beyond the scope of this paper why humans have an aptitude for this kind of visual processing - the kind that is leveraged for games of spot the difference. But the visual recognition of the difference is, in any case, only a first step. The inference from contrasts to causal relationships depends on a great deal of background knowledge, as the cases discussed here make plain. For a difference on an electrophoresis gel to be interpreted causally, it is necessary to know about the details of the experimental setup, of the interventions performed, and of the measurements taken in order to produce the figure.

The role of causal contrast diagrams goes beyond making patterns in the data salient to facilitate inferences: causal contrasts also have evidential roles. This is particularly striking in cases of detection such as in section 2.1 on post-transcriptional gene silencing, where images of an electrophoresis gel and of fluorescent proteins constitute part of the products of the experiments. Without these images, part of the evidential basis for the paper's causal claim would simply be lacking. By contrast, in the ecological case of section 2.2 and in the epidemiological case of section 2.3, the evidential role of the diagram is more indirect. But it is equally important: We saw that Gause's diagrams represent experimental data on population sizes at different time points. This data is either not presented in the text at all, or it is relegated to an (incomplete) appendix. Similarly, the diagram in the 1954 paper by Doll and Hill contains raw empirical values - actual numbers of deaths according to smoking severity and disease outcome that no other part of the paper provides.

As methods and technologies evolve, we should expect to see both continuity and change in the evidential roles of diagrams. ${ }^{12}$ On the one hand, the ubiquitous diagrams of visualized macromolecules in the life sciences retain their evidential function even though imaging has transitioned from analog to digital techniques. On the other hand, we would not expect modern diagrams to function quite like those of Gause or Doll and Hill since many leading journals now have data deposition policies (especially for clinical research) that mandate the sharing of full electronic data sets. In such cases, the data sets rather than the visuals become the basic, "raw" evidence available to readers. At the same time, however, technological change may also strengthen the evidential roles of diagrams: Funding agencies are now investing in the development of new tools for analyzing and visualizing large data sets. Arguably, raw data sets cannot be fully exploited as evidence until there are ways, diagrammatic and non-diagrammatic, to extract relevant trends, comparisons and contrasts from them. ${ }^{13}$

Can we explain why all of these diagrams are so markedly different despite the

our eyespan, relying on an active eye to select and make contrasts rather than on bygone memories of images scattered over pages and pages" (Tufte, 1990, p. 33). William C. Wimsatt (1990) has previously applied this approach in the philosophy of biology. I thank an anonymous referee for highlighting this connection.

${ }^{12}$ I thank an anonymous referee for drawing my attention to this point.

${ }^{13}$ See the press release "Obama Administration Unveils "Big Data" Initiative: Announces \$200 Million in new R\&D Investments", issued by the US Office of Science and Technology Policy on March 29, 2012. Tellingly, calls for "analysis" and "visualization" go largely hand in hand. 
conceptual unity provided by the identification of causal contrasts? The cases themselves suggest an answer. Scientists use modes of representation that fit the data being produced: the abundance of size-separated macromolecules (electrophoresis gel), the expression of reporter genes (fluorescence), the temporal evolution of population sizes (line graphs), numbers of deaths (bar graphs), different morphologies depending on limb bud size (bifurcating arrows), and combinations of phenotypic traits in populations (state space). Just as bar graphs would be inappropriate for representing continuous change over time, bifurcating arrows in a line drawing would not be capable of capturing what matters about the detection of mRNA fragments of a particular size on an electrophoresis gel. It is not at all clear that a unified mode of representation would be desirable. One highly plausible candidate for such a unified presentation of causal information is certainly not fit for purpose: representing causal relationships in terms of nodes and edges, as is common in philosophical discussions such as Spirtes et al. (2000) or Woodward (2003b). Directed graphs are themselves a specialized tool for representing aspects of causation, in particular relata, relations and topologies. To the extent that directed graphs could become a widely used tool in science, it would be to abstract, summarize and convey the results of causal inferences rather than to identify, justify or communicate contrasts in actual empirical data. Thus, the thesis is that causal contrasts are expressed in terms of a broad variety data-appropriate modes of representation so that they can play all of their cognitive, communicative, epistemic and evidential roles in particular research contexts.

The point of the present paper is not that all or even most diagrams should be understood as highlighting difference-makers. The claim is only that such diagrams are widespread and that they form a robust and identifiable category, even though they appear in many different guises. Diagrams of causal contrasts should thus be part of any future theory of scientific diagrams. It is an open question what other types of diagrams should be recognized (along the lines of the initial taxonomy suggested by Bechtel and Abrahamsen, 2015), and how frequent each type will turn out to be in different scientific disciplines.

A promising avenue for further research is to chart the connections between diagrams of causal contrasts and other types of diagrams. In many scientific papers, diagrams to delineate phenomena are combined with diagrams to elucidate the causes of the phenomena. We have seen, for instance, that many diagrams show not only that $\mathrm{C}$ is a cause of $\mathrm{E}$, but also that $\mathrm{E}$ is a narrowly specified effect of $\mathrm{C}$ (e.g. the suppression of specific mRNAs in section 2.1 or the causation of a specific cancer in section 2.3). Thus, in these instances diagrams to elucidate effects also contribute to specifying the phenomenon to be explained in the first place. Moreover, research papers are often focused on causal contrast diagrams but end with a diagram of a mechanism sketch. It is worthwhile to ask how these two types of diagrams interact, that is, how the knowledge gained from causal contrasts is integrated in mechanism diagrams. Conversely, we can 
ask how mechanism schemas motivate further empirical work on causal contrasts.

Studying the interaction of different types of diagrams will link questions about diagrams with traditional concerns in history and philosophy of science. For instance, we can analyze the role of diagrams in scientific discovery, in particular their role in defining and exploring the space of possible hypotheses to explain a phenomenon (see for instance Burnston, 2013; Scholl and Nickelsen, 2015). Similarly, causal contrast diagrams have obvious links to debates about causation and causal inference and thus to issues of evidence, confirmation and explanation - as explored, in part, in the paper in hand. Finally, the interaction of causal contrast diagrams and evolving mechanism diagrams may offer new insight into the character and causes of medium-term change in scientific concepts and hypotheses. An important step in this direction was recently taken by a pioneering study by Burnston et al. (2014), who pursue the development of a mechanistic diagram through multiple stages and relate its changes to diagrams of explanatory or causal relationships.

Biological diagrams are known to facilitate both communication and reasoning, to delineate phenomena, and to represent mechanisms. In addition, we have seen that some diagrams represent causal contrasts and are thus intimately linked to scientific epistemology: they play a key role both as representations of and as evidence for causal claims. It will be the task of a philosophy of scientific diagrams to refine and expand our understanding of why biology is such an emphatically visual science.

\section{References}

Alberch, P. (1982). Developmental constraints in evolutionary processes. In Bonner, J. T., editor, Evolution and development, pages 313-332. Springer.

Alberch, P. and Gale, E. A. (1985). A developmental analysis of an evolutionary trend: digital reduction in amphibians. Evolution, 39(1):8-23.

Bechtel, W. and Abrahamsen, A. (2012). Diagramming phenomena for mechanistic explanation. In Proceedings of the 34th annual conference of the Cognitive Science Society, pages 102-107. Cognitive Science Society, Austin, TX.

Bechtel, W. and Abrahamsen, A. (2015). Diagrams as tools for scientific reasoning. Review of Psychology and Philosophy, 6:117-131.

Bechtel, W., Burnston, D., Sheredos, B., and Abrahamsen, A. (2014). Representing time in scientific diagrams. In Proceeding of the 36th Annual Conference of the Cognitive Science Society, pages 164-169. Cognitive Science Society, Austin, TX.

Burnston, D. C. (2013). Mechanistic diagrams as search organizers. In Proceedings of the 35th Annual Conference of the Cognitive Science Society, pages 1952-1957. Cognitive Science Society, Austin, TX. 
Burnston, D. C. (2016). Data graphs and mechanistic explanation. Studies in History and Philosophy of Science Part C: Studies in History and Philosophy of Biological and Biomedical Sciences, 57:1-12.

Burnston, D. C., Sheredos, B., Abrahamsen, A., and Bechtel, W. (2014). Scientists use of diagrams in developing mechanistic explanations: A case study from chronobiology. Pragmatics \& Cognition, 22(2):224-243.

Craver, C. F. and Darden, L. (2013). In search of mechanisms: Discoveries across the life sciences. University of Chicago Press.

Crowley, T. E., Nellen, W., Gomer, R. H., and Firtel, R. A. (1985). Phenocopy of discoidin I-minus mutants by antisense transformation in Dictyostelium. Cell, 43(3):633-641.

Doll, R. and Hill, A. B. (1950). Smoking and carcinoma of the lung. British Medical Journal, 2(4682):739-748.

Doll, R. and Hill, A. B. (1952). Study of the aetiology of carcinoma of the lung. British Medical Journal, 2(4797):1271.

Doll, R. and Hill, A. B. (1954). The mortality of doctors in relation to their smoking habits. British Medical Journal, 1(4877):1451-1455.

Fire, A., Xu, S., Montgomery, M. K., Kostas, S. A., Driver, S. E., and Mello, C. C. (1998). Potent and specific genetic interference by double-stranded rna in caenorhabditis elegans. Nature, 391(6669):806-811.

Friendly, M. (2008). A brief history of data visualization. In Chen, C. H., Härdle, W., and Unwin, A., editors, Handbook of Data Visualization, pages 15-56. Springer.

Funkhouser, H. G. (1937). Historical development of the graphical representation of statistical data. Osiris, 3:269-404.

Gause, G. (1935). Experimental demonstration of Volterra's periodic oscillations in the numbers of animals. Journal of Experimental Biology, 12(1):44-48.

Gause, G. F. (1934a). Experimental analysis of Vito Volterra's mathematical theory of the struggle for existence. Science, 79(2036):16-17.

Gause, G. F. (1934b). The struggle for existence. Williams \& Wilkins.

Goodwin, W. (2009). Visual representations in science. Philosophy of Science, 76(3):372-390.

Gould, S. J. (2002). The structure of evolutionary theory. Harvard University Press. 
Hammond, S. M., Bernstein, E., Beach, D., and Hannon, G. J. (2000). An rna-directed nuclease mediates post-transcriptional gene silencing in drosophila cells. nature, 404(6775):293-296.

Hannon, G. J. and Rossi, J. J. (2004). Unlocking the potential of the human genome with rna interference. Nature, 431(7006):371-378.

Hegarty, M. (2004). Mechanical reasoning by mental simulation. Trends in cognitive sciences, 8(6):280-285.

Hegarty, M. (2011). The cognitive science of visual-spatial displays: Implications for design. Topics in cognitive science, 3(3):446-474.

Hegarty, M., Just, M. A., and Morrison, I. R. (1988). Mental models of mechanical systems: Individual differences in qualitative and quantitative reasoning. Cognitive Psychology, 20(2):191-236.

Hitchcock, C. (2012). Probabilistic causation. In Zalta, E. N., editor, The Stanford Encyclopedia of Philosophy. Winter 2012 edition.

Larkin, J. H. and Simon, H. A. (1987). Why a diagram is (sometimes) worth ten thousand words. Cognitive science, 11(1):65-100.

Lewis, D. (1974). Causation. The journal of philosophy, 70(17):556-567.

Lotka, A. J. et al. (1925). Elements of physical biology. Williams \& Wilkins.

Mill, J. S. (1843). A System of Logic. John W. Parker, London.

Perini, L. (2005a). Explanation in two dimensions: Diagrams and biological explanation. Biology and Philosophy, 20(2-3):257-269.

Perini, L. (2005b). The truth in pictures. Philosophy of Science, 72(1):262-285.

Perini, L. (2005c). Visual representations and confirmation. Philosophy of Science, 72(5):913-926.

Perini, L. (2012). Truth-bearers or truth-makers? Spontaneous Generations: A Journal for the History and Philosophy of Science, 6(1):142-147.

Scheines, R. (2005). The similarity of causal inference in experimental and nonexperimental studies. Philosophy of Science, 72(5):927-940.

Scholl, R. (2013). Causal inference, mechanisms, and the Semmelweis case. Studies in History and Philosophy of Science Part A, 44(1):66-76. 
Scholl, R. and Nickelsen, K. (2015). Discovery of causal mechanisms: Oxidative phosphorylation and the Calvin-Benson cycle. History and philosophy of the life sciences, 37(2):180-209.

Semmelweis, I. P. (1861). Die Aetiologie, der Begriff und die Prophylaxis des Kindbettfiebers. C. A. Hartleben, Pest, Wien and Leipzig.

Sheredos, B., Burnston, D., Abrahamsen, A., and Bechtel, W. (2013). Why do biologists use so many diagrams? Philosophy of Science, 80(5):931-944.

Spirtes, P., Glymour, C. N., and Scheines, R. (2000). Causation, prediction, and search, volume 81. MIT press.

Tufte, E. R. (1983). The visual display of quantitative information. Graphics Press Cheshire, CT.

Tufte, E. R. (1990). Envisioning Information. Graphics Press, Cheshire, CT.

Volterra, V. (1926). Fluctuations in the abundance of a species considered mathematically. Nature, 118(2972):558-560.

Volterra, V. (1928). Variations and fluctuations of the number of individuals in animal species living together. J. Cons. int. Explor. Mer, 3(1):3-51.

Waters, C. K. (2007). Causes that make a difference. The Journal of Philosophy, 104(11):551-579.

Wimsatt, W. C. (1990). Taming the dimensions-visualizations in science. In PSA: Proceedings of the Biennial Meeting of the Philosophy of Science Association, pages 111-135.

Woodward, J. (2003a). Experimentation, causal inference, and instrumental realism. In Radder, H., editor, The philosophy of scientific experimentation, chapter 5, pages 87-118. University of Pittsburgh Press, Pittsburgh, PA.

Woodward, J. (2003b). Making things happen: A theory of causal explanation. Oxford University Press.

Woodward, J. (2010). Causation in biology: stability, specificity, and the choice of levels of explanation. Biology \& Philosophy, 25(3):287-318.

Woodward, J. (2013). Causation and manipulability. In Zalta, E. N., editor, The Stanford Encyclopedia of Philosophy. Winter 2013 edition.

Wunderlich, K. R. A. (1868). Das Verhalten der Eigenwärme in Krankheiten. Otto Wigard, Leipzig. 